\title{
Article \\ The Optimal Control Problems for Generalized Elliptic Quasivariational Inequalities
}

\author{
Shih-Sen Chang ${ }^{1, *}$, Abdullah Ali H. Ahmadini ${ }^{2}{ }^{(D}$, Salahuddin ${ }^{2}{ }^{(0)}$, Min Liu ${ }^{3}$ and Jinfang Tang ${ }^{3}$ \\ 1 Center for General Education, China Medical University, Taichung 40402, Taiwan \\ 2 Department of Mathematics, Jazan University, Jazan 45142, Saudi Arabia; \\ aahmadini@jazanu.edu.sa (A.A.H.A.); dr_salah12@yahoo.com (S.) \\ 3 Department of Mathematics, Yibin University, Yibin 644007, China; liuminybsc@163.com (M.L.); \\ jinfangt_79@163.com (J.T.) \\ * Correspondence: changss2013@163.com
}

Citation: Chang, S.-S.; Ahmadini, A.A.H.; Salahuddin; Liu, M.; Tang, J. The Optimal Control Problems for Generalized Elliptic Quasivariational Inequalities. Symmetry 2022, 14, 199 https://doi.org/10.3390/ sym14020199

Academic Editors: Garik Petrosyan and Alexander Zaslavski

Received: 20 December 2021

Accepted: 13 January 2022

Published: 20 January 2022

Publisher's Note: MDPI stays neutral with regard to jurisdictional claims in published maps and institutional affiliations.

Copyright: (C) 2022 by the authors. Licensee MDPI, Basel, Switzerland. This article is an open access article distributed under the terms and conditions of the Creative Commons Attribution (CC BY) license (https:// creativecommons.org/licenses/by/ $4.0 /)$.

\begin{abstract}
In this article, we propose an optimal control problem for generalized elliptic quasivariational inequality with unilateral constraints. Then, we discuss the sufficient assumptions that ensure the convergence of the solutions to the optimal control problem. The proofs depend on convergence results for generalized elliptic quasi-variational inequalities, obtained by the arguments of compactness, lower semi-continuity, monotonicity, penalty and different estimates. As an application, we addressed the abstract convergence results in the analysis of optimal control associated with boundary value problems.
\end{abstract}

Keywords: convergence results; frictional contact; heat transfer; optimal control; optimal pair; generalized elliptic quasivariational inequalities; unilateral constraint

MSC: 49J27; 49K20; 74M15; 74M10; 49J40; 47H09; 47J20; 54H25; 49J53; 58E35; 35J66

\section{Introduction}

Many applications of optimal control theory can be found in physics, mechanics, automatics, systems theory, and financial management control theory. Although the control problems for linear systems are sufficiently well-studied, the situation is not so good for nonlinear systems. However, due to the complexity of nonlinear systems describing fluid motion and non-Newtonian fluid motion, such as polymers, various solutions, emulsions, blood, and many others, they have not been fully studied. In hydrodynamics, control (optimal) problems are often connected with fluid control by external forces. Usually, in solving such issues, a control is considered from a given finite set, see [1-5].

The concept of variational inequality was developed on the basis of monotonicity and convexity, including properties of the subdifferential of a convex function, see [6-10]. The study of optimal control problems for variational and hemivariational inequalities has been addressed in several works and is an expanding and vibrant branch of applied mathematics with numerous applications, see [11-17]. The theory and computational techniques for optimal control for equations and variational inequalities have been studied for quite some time now. In [18-21], the optimal control problem in the sense of boundary valued problems can be found in [22-26], and the computational issues have been addressed in [27-31]. Nonetheless, many important applied models have motivated the study of optimal control for more general quasi-variational problems.

In this paper, we consider an optimal control problem for a general class of elliptic quasivariational inequalities. 
Let $\mathcal{X}$ and $\mathcal{Y}$ be real Hilbert spaces endowed with the inner products $\langle\cdot, \cdot\rangle_{\mathcal{X}}$ and $\langle\cdot, \cdot\rangle_{\mathcal{Y}}$ respectively, $\mathscr{K} \subset \mathcal{X}, \mathscr{N}: \mathcal{X} \times \mathcal{X} \longrightarrow \mathcal{X}, J: \mathcal{X} \times \mathcal{X} \longrightarrow \mathbb{R}, \gamma: \mathcal{X} \longrightarrow \mathcal{Y}, f \in \mathcal{Y}$. Then, we consider the following inequality problem for finding $x \in \mathscr{K}$ such that

$$
\langle\mathscr{N}(x, x), y-x\rangle_{\mathcal{X}}+j(x, y)-j(x, x) \geq\langle f, \gamma y-\gamma x\rangle_{\mathcal{Y}}, \forall y \in \mathscr{K},
$$

and the admissible pairs set defined by

$$
\mathcal{V}_{a d}=\{(x, f) \in \mathscr{K} \times \mathcal{Y} \text { such that (1) holds }\}
$$

and we consider a objective functional $\mathscr{L}: \mathcal{X} \times \mathcal{Y} \longrightarrow \mathbb{R}$. Here and below, $\mathcal{X} \times \mathcal{Y}$ represents the product of the Hilbert spaces $\mathcal{X}$ and $\mathcal{Y}$, equipped with the canonical inner product. Then, we study, in this paper, the following optimal control problem for finding $\left(x^{*}, f^{*}\right) \in \mathcal{V}_{a d}$ such that

$$
\mathscr{L}\left(x^{*}, f^{*}\right)=\min _{(x, f) \in \mathcal{V}_{a d}} \mathscr{L}(x, f) .
$$

Next, consider a set $\hat{\mathscr{K}} \subset \mathcal{X}$, an operator $\hat{\mathscr{N}}: \mathcal{X} \times \mathcal{X} \longrightarrow \mathcal{X}$ and an element $\hat{f} \in \mathcal{Y}$. With these data, we suggest the following perturbation of (1) for finding $\hat{x} \in \mathscr{\mathscr { K }}$ such that

$$
\langle\hat{\mathscr{N}}(\hat{x}, \hat{x}), y-\hat{x}\rangle_{\mathcal{X}}+j(\hat{x}, y)-j(\hat{x}, \hat{x}) \geq\langle\hat{f}, \gamma y-\gamma \hat{x}\rangle_{\mathcal{Y}}, \forall y \in \hat{\mathscr{K}} .
$$

We associate to (4) the admissible pairs set given by

$$
\hat{\mathcal{V}}_{a d}=\{(\hat{x}, \hat{f}) \in \hat{\mathscr{K}} \times \mathcal{Y} \text { such that (4) holds }\}
$$

and, for a objective functional $\hat{\mathscr{L}}: \mathcal{X} \times \mathcal{Y} \longrightarrow \mathbb{R}$, we construct the following perturbation of the optimal control problem (3) for finding $\left(\hat{x}^{*}, \hat{f}^{*}\right) \in \hat{\mathcal{V}}_{a d}$ such that

$$
\hat{\mathscr{L}}\left(\hat{x}^{*}, \hat{f}^{*}\right)=\min _{(\hat{x}, \hat{f}) \in \hat{\mathcal{V}}_{a d}} \hat{\mathscr{L}}(\hat{x}, \hat{f}) .
$$

The unique solvability of (1) and (4), and the solvability of (3) and (6) follow from well known results obtained in the literature, under sufficient assumptions on the data. Here, we shall use the existence and uniqueness results in [32-35], which will be resumed in the next section.

The first goal of this paper is to formulate adequate assumptions on the data which guarantee the convergence of the solution $\hat{x}$ of (4) to the solution $x$ of (1). The second goal is to demonstrate that, under the suitable circumstances, the solutions of (6) converge to a solution of (3). Finally, we investigate and describe the applications in contact mechanics and a heat transfer process.

\section{Preliminaries}

Throughout the text" $\rightarrow$ " and " $\rightarrow$ " stand for the weak and the strong convergence, respectively. We denote by $\|\cdot\|_{\mathcal{X}}$ and $\|\cdot\|_{\mathcal{Y}}$, the norms on the spaces $\mathcal{X}$ and $\mathcal{Y}$, respectively. In our study of (1) we consider the following assumptions into the account:

(i)

$$
\mathscr{K} \text { is a nonempty, closed, convex subset of } \mathcal{X} \text {. }
$$

(ii) $\mathscr{N}$ is relaxed monotone with respect to the first variables if there exist $\alpha_{\mathscr{N}}>0$ such that

$$
\langle\mathscr{N}(x, x)-\mathscr{N}(y, y), x-y\rangle_{\mathcal{X}} \geq-\alpha_{\mathscr{N}}\|x-y\|_{\mathcal{X}}^{2}, \forall x, y \in \mathcal{X} .
$$


(iii) $\mathscr{N}$ is relaxed Lipschitz with respect to the second variables if there exist $\beta_{\mathscr{N}} \geq 0$ such that

$$
\langle\mathscr{N}(x, x)-\mathscr{N}(y, y), x-y\rangle_{\mathcal{X}} \leq-\beta_{\mathscr{N}}\|x-y\|_{\mathcal{X}}^{2}, \forall x, y \in \mathcal{X} .
$$

(iv) $\mathscr{N}$ is Lipschitz continuous if there exist constants $\xi_{\mathscr{N}}>0$ and $\varsigma_{\mathscr{N}}>$ such that

$$
\|\mathscr{N}(x, x)-\mathscr{N}(y, y)\| \leq \xi_{\mathscr{N}}\|x-y\|_{\mathcal{X}}+\varsigma_{\mathscr{N}}\|x-y\|_{\mathcal{X}}, \forall x, y \in \mathcal{X} .
$$

(v)

$$
\text { For all } \eta \in \mathcal{X}, \jmath(\eta, \cdot): \mathcal{X} \longrightarrow \mathbb{R} \text { is convex and lower semicontinuous (1.s.c), }
$$

(vi) There exists $\alpha_{j} \geq 0$ such that

$$
\jmath\left(\eta_{1}, y_{2}\right)-\jmath\left(\eta_{1}, y_{1}\right)+\jmath\left(\eta_{2}, y_{1}\right)-\jmath\left(\eta_{2}, y_{2}\right) \leq \alpha_{\jmath}\left\|\eta_{1}-\eta_{2}\right\| \mathcal{X}\left\|y_{1}-y_{2}\right\|_{\mathcal{X}}, \forall \eta_{1}, \eta_{2}, y_{1}, y_{2} \in \mathcal{X} \text {. }
$$

$$
\beta_{\mathscr{N}}-\alpha_{\mathscr{N}}>\alpha_{j}
$$

$$
f \in \mathcal{Y} .
$$

(ix) $\gamma$ is a linear continuous operator, i.e., there exists $\varrho_{0}>0$ such that

$$
\|\gamma y\|_{\mathcal{Y}} \leq \varrho_{0}\|y\|_{\mathcal{X}}, \forall y \in \mathcal{X} .
$$

Theorem 1 ([36]). Assume that (7)-(15) hold. Then, the quasi variational inequality (1) has a unique solution.

In the study of optimal control Problem, we assume that

$$
\mathscr{L}(x, f)=\mathscr{B}(x)+\mathscr{D}(f), \forall x \in \mathcal{X}, f \in \mathcal{Y},
$$

where $\mathscr{B}: \mathcal{X} \longrightarrow \mathbb{R}$ is continuous, positive and bounded, i.e.,

$$
\begin{gathered}
y_{n} \longrightarrow y \in \mathcal{X}, \quad \mathscr{B}\left(y_{n}\right) \longrightarrow \mathscr{B}(y) ; \\
\mathscr{B}(y) \geq 0, \forall y \in \mathcal{X} ;
\end{gathered}
$$

the mapping $\mathscr{B}$ is bounded sets in $\mathcal{X}$ into bounded sets in $\mathbb{R}$;

and $\mathscr{D}: \mathcal{Y} \longrightarrow \mathbb{R}$ is weakly lower semicontinuous and coercive, i.e.,

$$
\begin{gathered}
f_{n}-f \in \mathcal{Y} \quad \Rightarrow \liminf \mathscr{D}\left(f_{n}\right) \geq \mathscr{D}(f) ; \\
\left\|f_{n}\right\|_{\mathcal{Y}} \longrightarrow \infty \Rightarrow \mathscr{D}\left(f_{n}\right) \rightarrow \infty,
\end{gathered}
$$

also there exist $\lambda \geq 0, \rho \geq 0$ such that

$$
\begin{gathered}
\jmath\left(\eta, y_{1}\right)-\jmath\left(\eta, y_{2}\right) \leq\left(\lambda+\rho\|\eta\|_{\mathcal{X}}\right)\left\|y_{1}-y_{2}\right\|_{\mathcal{X}}, \forall \eta, y_{1}, y_{2} \in \mathcal{X}, \\
\beta_{\mathscr{N}}-\alpha_{\mathscr{N}}>\rho .
\end{gathered}
$$

For any sequences $\left\{\eta_{n}\right\} \subset \mathcal{X},\left\{x_{n}\right\} \subset \mathcal{X}$ such that

$$
\eta_{n} \rightarrow \eta \in \mathcal{X}, x_{n} \rightarrow x \in \mathcal{X}
$$

one has

$$
\limsup \left(\jmath\left(\eta_{n}, y\right)-\jmath\left(\eta_{n}, x_{n}\right)\right) \leq \jmath(\eta, y)-\jmath(\eta, x) \quad \forall y \in \mathcal{X} ;
$$


For any sequence $\left\{y_{n}\right\} \subset \mathcal{X}$ such that

$$
y_{n} \rightarrow y \in \mathcal{X} \text { one has } \gamma y_{n} \rightarrow \gamma y \in \mathcal{Y} .
$$

Theorem 2 ([36]). Assume that (7)-(26) holds. Then, there exists at least one solution

$$
\left(x^{*}, f^{*}\right) \in \mathcal{V}_{a d}
$$

of (3).

\section{Main Results}

In this section, we state and prove a convergence result for the solution of (4), in the case where the problem has a dynamical structure. To the end, we consider two sequences $\left\{\zeta_{n}\right\} \subset \mathbb{R},\left\{f_{n}\right\} \subset \mathcal{Y}$ and an operator $\mathscr{G}: \mathcal{X} \times \mathcal{X} \longrightarrow \mathcal{X}$. For each $n \in \mathbb{N}$ let $\mathscr{N}_{n}:$ $\mathcal{X} \times \mathcal{X} \longrightarrow \mathcal{X}$ be the operator defined by

$$
\mathscr{N}_{n}(x, x)=\mathscr{N}(x, x)+\frac{1}{\zeta_{n}} \mathscr{G}(x, x), \forall x \in \mathcal{X} .
$$

Assume that if $\hat{\mathscr{N}}=\mathscr{N}_{n}$ and $\hat{f}=f_{n}$, then we have a problem for finding $x_{n} \in \hat{\mathscr{K}}$ such that

$$
\left\langle\mathscr{N}\left(x_{n}, x_{n}\right), y-x_{n}\right\rangle \mathcal{X}+\frac{1}{\zeta_{n}}\left\langle\mathscr{G}\left(x_{n}, x_{n}\right), y-x_{n}\right\rangle \mathcal{X}+\jmath\left(x_{n}, y\right)-\jmath\left(x_{n}, x_{n}\right) \geq\left\langle f_{n}, \gamma y-\gamma x_{n}\right\rangle \mathcal{Y}, \forall y \in \hat{\mathscr{K}}
$$

Remark 1. $\hat{\mathscr{K}}=\mathcal{X}$ then (28) denotes the penalty problem of (1), under the suitable assumptions of $\mathscr{G}$, see $[37,38]$.

To demonstrate the solvability of (28), we have the following observations:

$$
\hat{\mathscr{K}} \text { is a nonempty, closed, convex subset of } \mathcal{X} \text {. }
$$

$\mathscr{G}: \mathcal{X} \times \mathcal{X} \longrightarrow \mathcal{X}$ is a relaxed monotone, relaxed Lipschitz and Lipschitz continuous operator.

$$
\begin{aligned}
& \zeta_{n}>0, \quad \forall n \in \mathbb{N} . \\
& f_{n} \in \mathcal{Y}, \quad \forall n \in \mathbb{N} .
\end{aligned}
$$

Lemma 1 ([39]). Assume (8)-(13), (15), (29)-(32). Then for each $n \in \mathbb{N}$, there exists a unique solution $x_{n} \in \mathcal{X}$ to (28).

To investigate the behavior of the solution of (28) as $n \longrightarrow \infty$ we offer the following additional assumptions.

$$
\begin{gathered}
\zeta_{n} \longrightarrow 0 \text { as } n \longrightarrow \infty . \\
f_{n} \rightarrow f \in \mathcal{Y} \text { as } n \longrightarrow \infty . \\
\mathscr{K} \subset \hat{\mathscr{K}} . \\
\langle\mathscr{G}(x, x), y-x\rangle_{\mathcal{X}}, \forall x \in \hat{\mathscr{K}}, y \in \mathscr{K},
\end{gathered}
$$

and

$$
x \in \hat{\mathscr{K}},\langle\mathscr{G}(x, x), y-x\rangle_{\mathcal{X}}=0, \forall y \in \mathscr{K} \Rightarrow x \in \mathscr{K}
$$

Remark 2. When $\hat{\mathscr{K}}=\mathcal{X}$, condition (36)-(37) is satisfied for any penalty operator of the set $\mathscr{K}$, see [40]. 
Theorem 3. Assume (7)-(15), (22)-(26), (29)-(37) and, for each $n \in \mathbb{N}$, denote by $x_{n}$ the solution of (28). Then

$$
x_{n} \longrightarrow x \in \mathcal{X}, \quad \text { as } n \longrightarrow \infty \text {, }
$$

where $x$ is the solution of (1).

Proof. There are several steps in the proof of the Theorem.

(i) The weak convergence.

We assert that there is an element $\hat{x} \in \hat{\mathscr{K}}$ and a subsequence of $\left\{x_{n}\right\}$, which is still denoted by $\left\{x_{n}\right\}$, such that

$$
x_{n} \rightarrow \hat{x} \in \mathcal{X} \text { as } n \longrightarrow \infty \text {. }
$$

To prove the claim, we establish the boundedness of the sequence $\left\{x_{n}\right\}$ in $\mathcal{X}$. Let $n \in \mathbb{N}$. We make use of the assumption (35) and take $y=x$ in (28) to see that

$\left\langle\mathscr{N}\left(x_{n}, x_{n}\right), x_{n}-x\right\rangle_{\mathcal{X}} \leq \frac{1}{\zeta_{n}}\left\langle\mathscr{G}\left(x_{n}, x_{n}\right), x-x_{n}\right\rangle_{\mathcal{X}}+\jmath\left(x_{n}, x\right)-\jmath\left(x_{n}, x_{n}\right)+\left\langle f_{n}, \gamma x_{n}-\gamma x\right\rangle_{\mathcal{Y}}$.

Then, using the relaxed monotonicity and relaxed Lipschitz continuity of the operator $\mathscr{N}$ we have

$$
\begin{aligned}
\left(\beta_{\mathscr{N}}-\alpha_{\mathscr{N}}\right)\left\|x_{n}-x\right\|_{\mathcal{X}}^{2} & \leq\left\langle\mathscr{N}(x, x), x-x_{n}\right\rangle_{\mathcal{X}}+\frac{1}{\zeta_{n}}\left\langle\mathscr{G}\left(x_{n}, x_{n}\right), x-x_{n}\right\rangle_{\mathcal{X}}+\jmath\left(x_{n}, x\right) \\
& -\jmath\left(x_{n}, x_{n}\right)+\left\langle f_{n}, \gamma x_{n}-\gamma x\right\rangle_{\mathcal{Y}} .
\end{aligned}
$$

Next, assumption (37) implies that

$$
\left\langle\mathscr{G}\left(x_{n}, x_{n}\right), x-x_{n}\right\rangle \mathcal{X} \leq 0
$$

and assumptions (11)-(12), (22) yield

$$
\begin{aligned}
\jmath\left(x_{n}, x\right)-\jmath\left(x_{n}, x_{n}\right) & =\left(\jmath\left(x_{n}, x\right)-\jmath\left(x_{n}, x_{n}\right)+\jmath\left(x, x_{n}\right)-\jmath(x, x)\right)+\left(\jmath(x, x)-\jmath\left(x, x_{n}\right)\right) \\
& \leq \alpha_{\jmath}\left\|x_{n}-x\right\|_{\mathcal{X}}^{2}+\left(\lambda+\rho\|x\|_{\mathcal{X}}\right)\left\|x_{n}-x\right\| \mathcal{X} .
\end{aligned}
$$

On the other side, using (15) we find that

$$
\left\langle\mathscr{N}(x, x), x-x_{n}\right\rangle_{\mathcal{X}}+\left\langle f_{n}, \gamma x_{n}-\gamma x\right\rangle_{\mathcal{Y}} \leq\left(\|\mathscr{N}(x, x)\|_{\mathcal{X}}+\varrho_{0}\left\|f_{n}\right\|_{\mathcal{Y}}\right)\left\|x_{n}-x\right\|_{\mathcal{X}}
$$

Now adding inequalities (38)-(41), we have

$$
\begin{aligned}
\left(\beta_{\mathscr{N}}-\alpha_{\mathscr{N}}\right)\left\|x_{n}-x\right\|_{\mathcal{X}}^{2} & \leq\left(\|\mathscr{N}(x, x)\|_{\mathcal{X}}+\varrho_{0}\left\|f_{n}\right\|_{\mathcal{Y}}\right)\left\|x_{n}-x\right\|_{\mathcal{X}}+\alpha_{j}\left\|x_{n}-x\right\|_{\mathcal{X}}^{2} \\
& +\left(\lambda+\rho\|x\|_{\mathcal{X}}\right)\left\|x_{n}-x\right\|_{\mathcal{X}} .
\end{aligned}
$$

From (34) we see that the sequence $\left\{f_{n}\right\}$ is bounded in $\mathcal{Y}$. Therefore, using inequality (42) and the smallness assumption (13), we deduce that there exists a constant $\vartheta>0$ independent of $n$ such that

$$
\left\|x_{n}-x\right\|_{\mathcal{X}} \leq \vartheta
$$

This implies that the sequence $\left\{x_{n}\right\}$ is bounded in $\mathcal{X}$. Thus, from the reflexivity of $\mathcal{X}$, we deduce that

$$
x_{n} \rightarrow \hat{x} \in \mathcal{X} \text {, as } n \longrightarrow \infty \text {, with some } \hat{x} \in \mathcal{X} \text {. }
$$

Moreover, assumption (29) and the convergence (43) implies that $\hat{x} \in \hat{\mathscr{K}}$ and the proof of assertion is completed.

(ii) The weak limit property.

Next, we show that $\hat{x}$ is a solution to (1).

Let $y$ be a given element in $\hat{\mathscr{K}}$ and let $n \in \mathbb{N}$. We use (28) to obtain that

$\frac{1}{\zeta_{n}}\left\langle\mathscr{G}\left(x_{n}, x_{n}\right), x_{n}-y\right\rangle_{\mathcal{X}} \leq\left\langle\mathscr{N}\left(x_{n}, x_{n}\right), y-x_{n}\right\rangle_{\mathcal{X}}+\jmath\left(x_{n}, y\right)-\jmath\left(x_{n}, x_{n}\right)+\left\langle f_{n}, \gamma x_{n}-\gamma y\right\rangle_{\mathcal{Y}}$. 
From the conditions (8)-(10), (34), (22), (15), and the boundedness of the sequence $\left\{x_{n}\right\}$, we see that each term in the right-hand side of the inequality (44) is bounded. Therefore, there exists a constant $\mathfrak{h}>0$ which does not depend on $n$, such that

$$
\left\langle\mathscr{G}\left(x_{n}, x_{n}\right), x_{n}-y\right\rangle \mathcal{X} \leq \zeta_{n} \mathfrak{h} .
$$

We now proceed to the upper limit in this inequality and use the convergence (33) to deduce that

$$
\limsup \left\langle\mathscr{G}\left(x_{n}, x_{n}\right), x_{n}-y\right\rangle \mathcal{X} \leq 0 .
$$

Next, we take $y=\hat{x}$ in (45) and find that

$$
\limsup \left\langle\mathscr{G}\left(x_{n}, x_{n}\right), x_{n}-\hat{x}\right\rangle_{\mathcal{X}} \leq 0 \text {. }
$$

Therefore, using assumption (30) and a standard pseudomonotonicity argument (Proposition 1.23 in $[38,41]$ ) we obtain that

$$
\liminf \left\langle\mathscr{G}\left(x_{n}, x_{n}\right), x_{n}-y\right\rangle_{\mathcal{X}} \geq\langle\mathscr{G}(\hat{x}, \hat{x}), \hat{x}-y\rangle_{\mathcal{X}}, \forall y \in \mathcal{X}
$$

We now combine the inequalities (47) and (45) to find that

$$
\langle\mathscr{G}(\hat{x}, \hat{x}), \hat{x}-y\rangle_{\mathcal{X}} \leq 0, \forall y \in \hat{\mathscr{K}} .
$$

Using the assumption (37), we can deduce that $\hat{x} \in \hat{\mathscr{K}}$. Now, consider an element $y \in \hat{\mathscr{K}}$. We use (35) and (28) to get

$$
\left\langle\mathscr{N}\left(x_{n}, x_{n}\right), x_{n}-y\right\rangle_{\mathcal{X}} \leq \frac{1}{\zeta_{n}}\left\langle\mathscr{G}\left(x_{n}, x_{n}\right), y-x_{n}\right\rangle_{\mathcal{X}}+\jmath\left(x_{n}, y\right)-\jmath\left(x_{n}, x_{n}\right)+\left\langle f_{n}, \gamma x_{n}-\gamma y\right\rangle \mathcal{Y} .
$$

Therefore, using assumption (36) we find that

$$
\left\langle\mathscr{N}\left(x_{n}, x_{n}\right), x_{n}-y\right\rangle_{\mathcal{X}} \leq \jmath\left(x_{n}, y\right)-\jmath\left(x_{n}, x_{n}\right)+\left\langle f_{n}, \gamma x_{n}-\gamma y\right\rangle_{\mathcal{Y}}
$$

Next, using (43) and assumption (25) we have

$$
\lim \sup \left(\jmath\left(x_{n}, y\right)-\jmath\left(x_{n}, x_{n}\right)\right) \leq \jmath(\hat{x}, y)-\jmath(\hat{x}, \hat{x}) .
$$

On the other side, assumption (34), (26) and the convergence (43) yield

$$
\left\langle f_{n}, \gamma x_{n}-\gamma y\right\rangle_{\mathcal{X}} \longrightarrow\langle f, \gamma \hat{x}-\gamma y\rangle_{\mathcal{Y}}
$$

Using the relations (48)-(50) to see that

$$
\limsup \left\langle\mathscr{N}\left(x_{n}, x_{n}\right), x_{n}-y\right\rangle_{\mathcal{X}} \leq \jmath(\hat{x}, y)-\jmath(\hat{x}, \hat{x})+\langle f, \gamma \hat{x}-\gamma y\rangle_{\mathcal{X}} .
$$

Now, taking $y=\hat{x}$ in (51) we get

$$
\limsup \left\langle\mathscr{N}\left(x_{n}, x_{n}\right), x_{n}-\hat{x}\right\rangle_{\mathcal{X}} \leq 0 .
$$

This inequality together with (43) and the pseudomonotonicity of $\mathscr{N}$ implies that

$$
\langle\mathscr{N}(\hat{x}, \hat{x}), \hat{x}-y\rangle_{\mathcal{X}} \leq \liminf \left\langle\mathscr{N}\left(x_{n}, x_{n}\right), x_{n}-y\right\rangle_{\mathcal{X}}, \forall y \in \mathcal{X} .
$$

Combining (53) and (51), we have

$$
\langle\mathscr{N}(\hat{x}, \hat{x}), \hat{x}-y\rangle_{\mathcal{X}} \leq \jmath(\hat{x}, y)-\jmath(\hat{x}, \hat{x})+\langle f, \gamma \hat{x}-\gamma y\rangle \mathcal{Y}, y \in \mathscr{K}
$$

Hence, it follows that $\hat{x} \in \mathscr{K}$ is a solution to (1), as claimed. 
(iii) Result of weak convergence.

Now, we prove that the whole sequence $\left\{x_{n}\right\}$ is weakly convergent. Since (1) has a unique solution $x \in \mathscr{K}$, we deduce from the previous step that $\hat{x}=x$. Moreover, a comprehensive review of the proof in step (ii) specifies that every subsequence of $\left\{x_{n}\right\}$ that converges weakly in $\mathcal{X}$ has the weak limit point $x$. We should also note that the sequence $\left\{x_{n}\right\}$ is bounded in $\mathcal{X}$. Therefore, using a standard argument we deduce that the whole sequence $\left\{x_{n}\right\}$ converges weakly in $\mathcal{X}$ to $x$, as $n \longrightarrow \infty$.

(iv) Strong convergence.

In the final step of the proof, we prove that

$$
x_{n} \longrightarrow x \in \mathcal{X} \text {, as } n \longrightarrow \infty .
$$

We take $y=\hat{x} \in \mathscr{K}$ in (53) and use (52) to obtain

$$
\begin{aligned}
0 & \leq \liminf \left\langle\mathscr{N}\left(x_{n}, x_{n}\right), x_{n}-\hat{x}\right\rangle_{\mathcal{X}} \\
& \leq \lim \sup \left\langle\mathscr{N}\left(x_{n}, x_{n}\right), x_{n}-\hat{x}\right\rangle_{\mathcal{X}} \\
& \leq 0
\end{aligned}
$$

which shows that

$$
\left\langle\mathscr{N}\left(x_{n}, x_{n}\right), x_{n}-\hat{x}\right\rangle_{\mathcal{X}} \longrightarrow 0 \text { as } n \longrightarrow \infty \text {. }
$$

Therefore, using equality $\hat{x}=x$, the relaxed monotonicity and relaxed Lipschitz continuity of $\mathscr{N}$, and the convergence

$$
x_{n} \rightarrow x \in \mathcal{X},
$$

we have

$$
\begin{aligned}
\left(\beta_{\mathscr{N}}-\alpha_{\mathscr{N}}\right)\left\|x_{n}-x\right\|_{\mathcal{X}}^{2} & \leq\left\langle\mathscr{N}\left(x_{n}, x_{n}\right)-\mathscr{N}(x, x), x_{n}-x\right\rangle_{\mathcal{X}} \\
& =\left\langle\mathscr{N}\left(x_{n}, x_{n}\right), x_{n}-x\right\rangle_{\mathcal{X}}-\left\langle\mathscr{N}(x, x), x_{n}-x\right\rangle_{\mathcal{X}} \longrightarrow 0 \text { as } n \longrightarrow \infty .
\end{aligned}
$$

Hence, it follows that $x_{n} \longrightarrow x \in \mathcal{X}$, which completes the proof.

\section{Optimal Control Analysis}

In this section, we connect an optimal control problem with (28) for which we prove a convergence result. To this end, we hold the previous section's notations and assumptions and define the set of admissible pairs for (28) by

$$
\mathcal{V}_{a d}^{n}=\left\{\left(x_{n}, f_{n}\right) \in \hat{\mathscr{K}} \times \mathcal{Y} \text { such that (28) holds }\right\} .
$$

Then, the optimal control problem associated to (28) is follows for finding $\left(x_{n}^{\star}, f_{n}^{\star}\right) \in \mathcal{V}_{a d}^{n}$ such that

$$
\mathscr{L}_{n}\left(x_{n}^{\star}, f_{n}^{\star}\right)=\min _{\left(x_{n}, f_{n}\right) \in \mathcal{V}_{a d}^{n}} \mathscr{L}_{n}\left(x_{n}, f_{n}\right) .
$$

In the study of (56), we assume that

$$
\mathscr{L}_{n}(x, f)=\mathscr{B}_{n}(x)+\mathscr{D}_{n}(f), \forall x \in \mathcal{X}, f \in \mathcal{Y},
$$

where $\mathscr{B}_{n}$ and $\mathscr{D}_{n}$ are functions which satisfy assumptions (17)-(19) and (20)-(21), for each $n \in \mathbb{N}$, respectively. Note that, when we use these assumptions for the functions $\mathscr{B}_{n}$ and $\mathscr{D}_{n}$, we refer to them as assumptions $(17)_{n}-(19)_{n}$ and $(20)_{n}-(21)_{n}$, respectively. Using Theorem 2, we have the following result. 
Lemma 2 ([39]). Assume that (8)-(13), (15), (57), (17) $n_{n}-(19)_{n},(20)_{n}-(21)_{n}$, (22)-(26) and (29)-(32) hold. Then, for each $n \in \mathbb{N}$, there exists at least one solution $\left(x_{n}^{\star}, f_{n}^{\star}\right) \in \mathcal{V}_{a d}^{n}$ of (56).

To study the behavior of the sequence of solutions of (56) as $n \longrightarrow \infty$ we consider the following additional hypotheses.

$$
\begin{gathered}
x_{n} \rightarrow x \in \mathcal{X} \Rightarrow \mathscr{B}_{n}\left(x_{n}\right) \rightarrow \mathscr{B}(x) . \\
f_{n} \rightarrow f \in \mathcal{Y} \Rightarrow \liminf \mathscr{D}_{n}\left(f_{n}\right) \geq \mathscr{D}(f) . \\
\left\|f_{n}\right\|_{\mathcal{Y}} \longrightarrow \infty \Rightarrow \mathscr{D}_{n}\left(f_{n}\right) \rightarrow \infty . \\
\mathscr{D}_{n}(f) \rightarrow \mathscr{D}(f), \quad \forall f \in \mathcal{Y} .
\end{gathered}
$$

Theorem 4. Assume that (7)-(13), (15), (26), (29)-(33), (35)-(37), (17) $n-(19)_{n},(20)_{n}-(21)_{n}$, and (57)-(61) hold and $\left\{\left(x_{n}^{\star}, f_{n}^{\star}\right)\right\}$ is a sequence of the solutions of problem (56). Then there exists a subsequence of the sequence $\left\{\left(x_{n}^{\star}, f_{n}^{\star}\right)\right\}$, again denoted by $\left\{\left(x_{n}^{\star}, f_{n}^{\star}\right)\right\}$, and an element $\left(x^{\star}, f^{\star}\right) \in \mathcal{X} \times \mathcal{Y}$ such that

$$
\begin{aligned}
& f_{n}^{\star} \rightarrow f^{\star} \in \mathcal{Y} \text { as } n \longrightarrow \infty, \\
& x_{n}^{\star} \rightarrow x^{\star} \in \mathcal{X} \text { as } n \longrightarrow \infty, \\
& \left(x^{\star}, f^{\star}\right) \text { is a solution of }(3)
\end{aligned}
$$

Proof. The proof is carried out in following manner.

(i) Boundedness.

We claim that the sequence $\left\{f_{n}^{\star}\right\}$ is bounded in $\mathcal{Y}$. Contrary we assume that $\left\{f_{n}^{\star}\right\}$ is not bounded in $\mathcal{Y}$, then passing to a subsequence still denoted $\left\{f_{n}^{\star}\right\}$, we have

$$
\left\|f_{n}^{\star}\right\|_{\mathcal{Y}} \longrightarrow+\infty \text { as } n \longrightarrow+\infty \text {. }
$$

By using the equality (57) and assumption (18) $)_{n}$, we have

$$
\mathscr{L}_{n}\left(x_{n}^{\star}, f_{n}^{\star}\right) \geq \mathscr{D}_{n}\left(f_{n}^{\star}\right)
$$

Therefore, passing to the limit as $n \longrightarrow \infty$ in this inequality and using (65) combined with assumption (60) we deduce that

$$
\lim \mathscr{L}_{n}\left(x_{n}^{\star}, f_{n}^{\star}\right)=+\infty
$$

On the other side, since $\left(x_{n}^{\star}, f_{n}^{\star}\right)$ represents a solution to (56) for each $n \in \mathbb{N}$ we have

$$
\mathscr{L}_{n}\left(x_{n}^{\star}, f_{n}^{\star}\right) \leq \mathscr{L}_{n}\left(x_{n}, f_{n}\right), \forall\left(x_{n}, f_{n}\right) \in \mathcal{V}_{a d}^{n} .
$$

We now denote by $x_{n}^{0}$ the solution of (28) for $f_{n}=f$. Then $\left(x_{n}^{0}, f\right) \in \mathcal{V}_{a d}^{n}$, and from (67) and (57) we have

$$
\mathscr{L}_{n}\left(x_{n}^{\star}, f_{n}^{\star}\right) \leq \mathscr{B}_{n}\left(x_{n}^{0}\right)+\mathscr{D}_{n}(f) .
$$

From (33) and (34) and Theorem 3 we have

$$
x_{n}^{0} \rightarrow x \in \mathcal{X} \text { as } n \longrightarrow \infty
$$

where $x$ represents the solution of (1). Then, assumptions (58) and (61) imply that

$$
\mathscr{B}_{n}\left(x_{n}^{0}\right)+\mathscr{D}_{n}(f) \rightarrow \mathscr{B}(x)+\mathscr{D}(f) .
$$

Relations (66), (68) and (70) lead to a contradiction, which concludes the claim. 
(ii) Convergence results.

In this step, we prove the convergence of the equations (62) and (63).

First, since the sequence $\left\{f_{n}^{\star}\right\}$ is bounded in $\mathcal{Y}$, there exists a subsequence again denoted by $\left\{f_{n}^{\star}\right\}$ and an element $f^{\star} \in \mathcal{Y}$ such that (62) holds.

Following that, $x^{\star}$ is a solution of (1) for $f=f^{\star}$. Then we have

$$
\left(x^{\star}, f^{\star}\right) \in \mathcal{V}_{a d} .
$$

Furthermore, assumption (33), the convergence (62) and Theorem 3 imply that (63) holds as well.

(iii) The limit of optimality.

We now prove that $\left(x^{\star}, f^{\star}\right)$ is a solution to the optimal control (3). We use the convergences (62), (63) and assumptions (58), (59), to see that

$$
\lim \inf \left(\mathscr{B}_{n}\left(x_{n}^{\star}\right)+\mathscr{D}_{n}\left(f_{n}^{\star}\right)\right) \geq \mathscr{B}\left(x^{\star}\right)+\mathscr{D}\left(f^{\star}\right)
$$

and, therefore, the structure (57) and (16) of the functionals $\mathscr{L}_{n}$ and $\mathscr{L}$ shows that

$$
\mathscr{L}\left(x^{\star}, f^{\star}\right) \leq \liminf \mathscr{L}_{n}\left(x_{n}^{\star}, f_{n}^{\star}\right) .
$$

Next, we fix a solution $\left(x_{0}^{\star}, f_{0}^{\star}\right)$ of (3) and, moreover, for each $n \in \mathbb{N}$ we denote by $\hat{x}_{n}^{0}$ the solution of (28) for $f_{n}=f_{0}^{\star}$. It follows from here that $\left(\hat{x}_{n}^{0}, f_{0}^{\star}\right) \in \mathcal{V}_{a d}^{n}$ and, by the optimality of the pair $\left(x_{n}^{\star}, f_{n}^{\star}\right)$, we have

$$
\mathscr{L}_{n}\left(x_{n}^{\star}, f_{n}^{\star}\right) \leq \mathscr{L}_{n}\left(\hat{x}_{n}^{0}, f_{0}^{\star}\right), \forall n \in \mathbb{N} .
$$

We proceed to the upper limit of this inequality to discover that

$$
\lim \sup \mathscr{L}_{n}\left(x_{n}^{\star}, f_{n}^{\star}\right) \leq \lim \sup \mathscr{L}_{n}\left(\hat{x}_{n}^{0}, f_{0}^{\star}\right) .
$$

Now, we know that $x_{0}^{\star}$ is the solution of the inequality (1) for $f=f_{0}^{\star}$ and $\hat{x}_{n}^{0}$ is the solution of the inequality (1) for $f_{n}=f_{0}^{\star}$. As a result, the convergence (33) and Theorem 3 imply that

$$
\hat{x}_{n}^{0} \longrightarrow x_{0}^{\star} \in \mathcal{X} \text { as } n \longrightarrow \infty
$$

and, using assumptions (58) and (61), we find that

$$
\mathscr{B}_{n}\left(\hat{x}_{n}^{0}\right) \longrightarrow \mathscr{B}_{n}\left(x_{0}^{\star}\right), \quad \mathscr{D}_{n}\left(f_{0}^{\star}\right) \longrightarrow \mathscr{D}\left(f_{0}^{\star}\right) \text { as } n \longrightarrow \infty \text {. }
$$

We now use (57), (74) and (16) to get

$$
\lim \mathscr{L}_{n}\left(\hat{x}_{n}^{0}, f_{0}^{\star}\right)=\lim \mathscr{L}\left(x_{0}^{\star}, f_{0}^{\star}\right) .
$$

Therefore, (72), (73) and (75) imply that

$$
\mathscr{L}\left(x^{\star}, f^{\star}\right) \leq \mathscr{L}\left(x_{0}^{\star}, f_{0}^{\star}\right) .
$$

On the other side, since $\left(x_{0}^{\star}, f_{0}^{\star}\right)$ is a solution of (3), we have

$$
\mathscr{L}\left(x_{0}^{\star}, f_{0}^{\star}\right)=\min _{(x, f) \in \mathcal{V}_{a d}} \mathscr{L}(x, f) .
$$

and, therefore, inclusion (71) implies that

$$
\mathscr{L}\left(x_{0}^{\star}, f_{0}^{\star}\right) \leq \mathscr{L}\left(x^{\star}, f^{\star}\right) .
$$

We now combine the inequalities (76) and (78) to see that

$$
\mathscr{L}\left(x^{\star}, f^{\star}\right)=\mathscr{L}\left(x_{0}^{\star}, f_{0}^{\star}\right) .
$$


Finally, relations (71), (79) and (77) imply that (64) holds and proof is completed.

\section{Optimal Control Associated with Frictional Contact Problem}

In this section, we will discuss the equilibrium of elastic bodies in a frictional contact problems, and in order to do so, we will require some notations and assumptions.

Let $d \in\{2,3\}$. Consider $\mathbb{S}^{d}$ to be a space of second order symmetric tensors on $\mathbb{R}^{d}$, and use the notation $\langle\cdot, \cdot\rangle,\|\cdot\|, \mathbf{0}$ to represent the inner product, norm, and zero element of the spaces $\mathbb{R}^{d}$ and $\mathbb{S}^{d}$, respectively. Let $\Omega \subset \mathbb{R}^{d}$ be a domain with a smooth boundary $\partial \Omega$ divided into three measurable disjoint parts $\left.\rceil_{1},\right\rceil_{2}$ and $\rceil_{3}$ with

$$
\left.\operatorname{meas}(\urcorner_{1}\right)>0 \text {. }
$$

A generic point in $\Omega \cup\urcorner$ will be denoted by $\mathbf{u}=\left(u_{i}\right)$ and $v=v_{i}$ represents the unit outward normal to 7 . We use the standard notation for Sobolev and Lebesgue spaces corresponding to $\Omega$ and $\urcorner$. In particular, we use the spaces $\left.\left.L^{2}(\Omega)^{d}, L^{2}(\urcorner_{2}\right)^{d}, L^{2}(\urcorner_{3}\right)$ and $H^{1}(\Omega)^{d}$, endowed with their canonical inner products and associated norms. Furthermore, for an element $v \in H^{1}(\Omega)^{d}$ we still write $v$ for the trace of $v$ to 7 . We also considered the space

$$
\left.\mathrm{V}=\left\{\boldsymbol{v} \in H^{1}(\Omega)^{d}: v=0 \text { on }\right\urcorner_{1}\right\},
$$

which is a real Hilbert space with canonical inner product

$$
\langle\mathbf{x}, \mathbf{y}\rangle_{\mathrm{V}}=\int_{\Omega} \varepsilon(\mathbf{x}) \cdot \varepsilon(\mathbf{y}) d x
$$

and the associated norm $\|\cdot\|_{\mathrm{V}}$. Here and below $\varepsilon$ represents the deformation operator, i.e.,

$$
\varepsilon(\mathbf{x})=\left(\varepsilon_{i j}(\mathbf{x})\right), \quad \varepsilon_{i j}(\mathbf{x})=\frac{1}{2}\left(x_{i, j}+x_{j, i}\right),
$$

where an index that follows a comma denotes the partial derivative with respect to the corresponding component of $\mathbf{u}$, e.g.,

$$
x_{i, j}=\frac{\partial x_{i}}{\partial j} .
$$

The assumption that meas (\urcorner$\left._{1}\right)>0$ allows us to apply Korn's inequality which results in the completeness of the space $\mathrm{V}$. We denote by $\mathbf{0}_{\mathrm{V}}$ the zero element of $\mathrm{V}$, and for an element $v \in \mathrm{V}$, the normal and tangential components on 7 are defined by

$$
v_{\mathrm{V}}=\boldsymbol{v} \cdot \boldsymbol{v} \text { and } \boldsymbol{v}_{\tau}=\boldsymbol{v}-\boldsymbol{v}_{\mathrm{V}} \boldsymbol{v}
$$

respectively. Recall the trace inequality

$$
\|\boldsymbol{v}\|_{L^{2}(\mathrm{~T})^{d}} \leq d_{0}\|\boldsymbol{v}\|_{\mathrm{V}}, \forall \boldsymbol{v} \in \mathrm{V},
$$

where $d_{0}$ denotes the positive constant. For the sake of convenience, we use the data $\mathscr{F}, p$, $\mathbf{f}_{0}, \mathbf{f}_{2}, \mu$ and $\kappa$ to satisfy the following conditions.

$$
\mathscr{F}: \mathbb{S}^{d} \times \mathbb{S}^{d} \longrightarrow \mathbb{S}^{d}
$$

There exist $\xi_{\mathscr{F}}>0$ and $\varsigma_{\mathscr{F}}$ such that

$$
\left\|\mathscr{F}\left(\varepsilon_{1}, \varepsilon_{1}\right)-\mathscr{F}\left(\varepsilon_{2}, \varepsilon_{2}\right)\right\| \leq \xi_{\mathscr{F}}\left\|\varepsilon_{1}-\varepsilon_{2}\right\|+\varsigma \mathscr{F}\left\|\varepsilon_{1}-\varepsilon_{2}\right\|, \quad \forall \varepsilon_{1}, \varepsilon_{2} \in \mathbb{S}^{d} .
$$


There exist $\alpha_{\mathscr{F}}>0$ and $\beta_{\mathscr{F}} \geq 0$ such that

$$
\begin{aligned}
&\left\langle\mathscr{F}\left(\varepsilon_{1}, \varepsilon_{1}\right)-\mathscr{F}\left(\varepsilon_{2}, \varepsilon_{2}\right), \varepsilon_{1}-\varepsilon_{2}\right\rangle \geq-\alpha_{\mathscr{F}}\left\|\varepsilon_{1}-\varepsilon_{2}\right\|^{2}, \forall \varepsilon_{1}, \varepsilon_{2} \in \mathbb{S}^{d}, \\
&\left\langle\mathscr{F}\left(\varepsilon_{1}, \varepsilon_{1}\right)-\mathscr{F}\left(\varepsilon_{2}, \varepsilon_{2}\right), \varepsilon_{1}-\varepsilon_{2}\right\rangle \leq-\beta \mathscr{F}\left\|\varepsilon_{1}-\varepsilon_{2}\right\|^{2}, \forall \varepsilon_{1}, \varepsilon_{2} \in \mathbb{S}^{d} . \\
& p: \mathbb{R} \longrightarrow \mathbb{R}_{+} .
\end{aligned}
$$

There exists $\xi_{p}>0$ such that

$$
\begin{gathered}
\left|p\left(r_{1}\right)-p\left(r_{2}\right)\right| \leq \xi_{p}\left|r_{1}-r_{2}\right|, \quad \forall r_{1}, r_{2} \in \mathbb{R} . \\
\left\langle p\left(r_{1}\right)-p\left(r_{2}\right), r_{1}-r_{2}\right\rangle \geq 0, \forall r_{1}, r_{2} \in \mathbb{R} . \\
p(r)=0 \Longleftrightarrow r \leq 0 . \\
\left.\mathbf{f}_{0} \in L^{2}(\Omega)^{d}, \mathbf{f}_{2} \in L^{2}(\urcorner_{2}\right)^{d} . \\
\mu>0 . \\
d_{0}^{2} \mu \xi_{p}<\beta_{\mathscr{F}}-\alpha \mathscr{F} . \\
\kappa>0 .
\end{gathered}
$$

Moreover, we use $\mathcal{Y}$ for the product space $\left.L^{2}(\Omega)^{d} \times L^{2}(\urcorner_{3}\right)^{d}$ equipped with the canonical inner product, and $\mathscr{K}$ for the set defined by

$$
\left.\mathscr{K}=\left\{\boldsymbol{v} \in \mathrm{V}: v_{v} \leq \kappa \text { a.e. on }\right\rceil_{3}\right\} .
$$

So there is the inequality problem we consider in order to find $\mathbf{x} \in \mathscr{K}$ such that

$$
\begin{aligned}
\int_{\Omega} \mathscr{F}(\varepsilon(\mathbf{x}), \varepsilon(\mathbf{x})) \cdot(\varepsilon(\mathbf{y})-\varepsilon(\mathbf{x})) d x+\int_{\urcorner_{3}} p\left(x_{v}\right)\left(y_{v}-x_{v}\right) d a & +\int_{\urcorner_{3}} \mu p\left(x_{v}\right)\left(\left\|\mathbf{y}_{\tau}\right\|-\left\|\mathbf{x}_{\tau}\right\|\right) d a \\
& \geq \int_{\Omega} \mathbf{f}_{0} \cdot(\mathbf{y}-\mathbf{x}) d x .
\end{aligned}
$$

Here, the elastic body $\Omega$, which is subjected to external forces, is fixed on $\urcorner_{1}$ and in frictional contact with $\rceil_{3}$. The contact takes place with a layer of deformable material of thickness $\kappa$. The elasticity operator is denoted by $\mathscr{F}$, and the density of applied body forces and traction acting on the body and the surface is denoted by $\mathbf{f}_{0}$ and $\mathbf{f}_{2}, 7_{2}$, respectively, $p$ is a given function that defines the deformable material's reaction, and $\mu$ represents the coefficient of friction. Next, we consider the constants $\pi_{0}, \pi_{2}, \pi_{3}$ and a function $\theta$ such that

$$
\left.\pi_{0}>0, \pi_{2}>0, \pi_{3}>0, \quad \theta \in L^{2}(\urcorner_{3}\right) .
$$

We associate to (94) the set of admissible pairs $\mathcal{V}_{a d}^{c}$ and the cost functional $\mathscr{L}$ given by

$$
\begin{array}{r}
\mathcal{V}_{a d}^{c}=\left\{(\mathbf{x}, \mathbf{f}) \in \mathscr{K} \times \mathcal{Y} \text { such that } \mathbf{f}=\left(\mathbf{f}_{0}, \mathbf{f}_{2}\right) \text { and (94) holds }\right\}, \\
\mathscr{L}(\mathbf{x}, \mathbf{f})=\pi_{0} \int_{\Omega}\left\|\mathbf{f}_{0}\right\|^{2} d x+\pi_{2} \int_{\urcorner_{2}}\left\|\mathbf{f}_{2}\right\|^{2} d a+\pi_{3} \int_{\urcorner_{3}}\left|x_{v}-\theta\right|^{2} d a, \forall \mathbf{x} \in \mathrm{V}, \mathbf{f}=\left(\mathbf{f}_{0}, \mathbf{f}_{2}\right) \in \mathcal{Y} .
\end{array}
$$

Furthermore, we consider the optimal control problem of finding $\left(\mathbf{x}^{\star}, \mathbf{f}^{\star}\right) \in \mathcal{V}_{a d}^{c}$ such that

$$
\mathscr{L}\left(\mathbf{x}^{\star}, \mathbf{f}^{\star}\right)=\min _{(\mathbf{x}, \mathbf{f}) \in \mathcal{V}_{a d}^{c}} \mathscr{L}(\mathbf{x}, \mathbf{f}) .
$$

Next, we take a look at a function $q$ and a constant $\hat{\kappa}$ satisfy the following conditions.

$$
q: \mathbb{R} \longrightarrow \mathbb{R}_{+} .
$$

There exists $\xi_{q}>0$ such that

$$
\left|q\left(r_{1}\right)-q\left(r_{2}\right)\right| \leq \xi_{q}\left|r_{1}-r_{2}\right|, \forall r_{1}, r_{2} \in \mathbb{R}
$$




$$
\begin{gathered}
\left\langle q\left(r_{1}\right)-q\left(r_{2}\right), r_{1}-r_{2}\right\rangle \geq 0, \forall r_{1}, r_{2} \in \mathbb{R} . \\
q(r)=0 \Longleftrightarrow r \leq 0 . \\
\hat{\kappa} \geq \kappa>0 .
\end{gathered}
$$

We introduce the set

$$
\left.\hat{\mathscr{K}}=\left\{\mathbf{y} \in \mathrm{V}: y_{v} \leq \hat{\kappa} \text { on }\right\urcorner_{3}\right\}
$$

and we assume that for each $n \in \mathbb{N}$ the functions $\mathbf{f}_{0 n}, \mathbf{f}_{2 n}, \theta_{n}$ and the constant $\zeta_{n}$ are given and satisfy the following conditions:

$$
\begin{gathered}
\left.\mathbf{f}_{0 n} \in L^{2}(\Omega)^{d}, \quad \mathbf{f}_{2 n} \in L^{2}(\urcorner_{3}\right)^{d}, \\
\left.\zeta_{n}>0, \quad \theta_{n} \in L^{2}(\urcorner_{3}\right) .
\end{gathered}
$$

Now, for each $n \in \mathbb{N}$, we consider the following perturbation of (94) for finding $\mathbf{x}_{n} \in \hat{\mathscr{K}}$ such that

$$
\begin{aligned}
& \int_{\Omega} \mathscr{F}\left(\varepsilon\left(\mathbf{x}_{n}\right), \varepsilon\left(\mathbf{x}_{n}\right)\right) \cdot\left(\varepsilon(\mathbf{y})-\varepsilon\left(\mathbf{x}_{n}\right)\right) d x+\int_{\urcorner_{3}} p\left(x_{n v}\right)\left(y_{v}-x_{n v}\right) d a+\frac{1}{\zeta_{n}} \int_{\urcorner_{3}} q\left(x_{n v}-\kappa\right)\left(y_{v}-x_{n v}\right) d a \\
& +\mu \int_{\urcorner_{3}} p\left(x_{n v}\right)\left(\left\|\mathbf{y}_{\tau}\right\|-\left\|\mathbf{x}_{n \tau}\right\|\right) d a \geq \int_{\Omega} \mathbf{f}_{0 n} \cdot(\mathbf{y}-\mathbf{x}) d x+\int_{\urcorner_{2}} \mathbf{f}_{2 n} \cdot\left(\mathbf{y}-\mathbf{x}_{n}\right) d a \forall \mathbf{y} \in \hat{\mathscr{K}} .
\end{aligned}
$$

The problem (107) is a variational formulation of the contact problem where the rigid body is covered by a layer of deformable material of thickness $\hat{\kappa}$. Here, the layer is divided into two parts: the first layer is located on the top of the rigid body with a thickness $\hat{\kappa}-\kappa>0$ and the second layer is located above with a thickness $\kappa$. Since $\zeta_{n}$ is the deformability coefficient of the first layer, therefore $\frac{1}{\zeta_{n}}$ denotes its stiffness coefficient, and $q$ is a normal compliance function of the first layer.

With the problem (107), we associate the set of admissible pairs $\mathcal{V}_{a d}^{c n}$ and the cost function $\mathscr{L}_{n}$ given by

$$
\begin{array}{r}
\mathcal{V}_{a d}^{c n}=\left\{\left(\mathbf{x}_{n}, \mathbf{f}_{n}\right) \in \hat{\mathscr{K}} \times \mathcal{Y} \text { such that } \mathbf{f}=\left(\mathbf{f}_{0 n}, \mathbf{f}_{2 n}\right) \text { and (107) holds }\right\}, \\
\mathscr{L}_{n}\left(\mathbf{x}_{n}, \mathbf{f}_{n}\right)=\pi_{0} \int_{\Omega}\left\|\mathbf{f}_{0 n}\right\|^{2} d x+\pi_{2} \int_{\urcorner_{2}}\left\|\mathbf{f}_{2 n}\right\|^{2} d a+\pi_{3} \int_{\urcorner_{3}}\left|x_{n v}-\theta_{n}\right|^{2} d a, \forall \mathbf{x}_{n} \in \mathrm{V}, \mathbf{f}_{n}=\left(\mathbf{f}_{0 n}, \mathbf{f}_{2 n}\right) \in \mathcal{Y} .
\end{array}
$$

Theorem 5. Assume that (82)-(91), (95), (99)-(103), (105) and (106) hold. Then

(a) (94) has a unique solution and, for each $n \in \mathbb{N}$ (107) has a unique solution. Furthermore, if

$$
\left.\zeta_{n} \longrightarrow 0, \mathbf{f}_{0 n} \rightarrow \mathbf{f}_{0} \in L^{2}(\Omega), \mathbf{f}_{2 n} \rightarrow \mathbf{f}_{2} \in L^{2}(\urcorner_{3}\right) \text { as } n \longrightarrow \infty,
$$

the solution of (107) converges to the solution of (94), i.e.,

$$
\mathbf{x}_{n} \rightarrow \mathbf{x} \in V \text { as } n \longrightarrow \infty \text {. }
$$

(b) (98) has at least one solution and, for each $n \in \mathbb{N}$, (109) has at least one solution. Moreover, if

$$
\left.\zeta_{n} \longrightarrow 0, \theta_{n} \longrightarrow \theta \in L^{2}(\urcorner_{3}\right) \text {, as } n \longrightarrow \infty,
$$

and $\left\{\left(\mathbf{x}_{n}^{\star}, \mathbf{f}_{n}^{\star}\right)\right\}$ is a sequence of solutions of (109), there exists a subsequence of the sequence $\left\{\left(\mathbf{x}_{n}^{\star}, \mathbf{f}_{n}^{\star}\right)\right\}$, again denoted by $\left\{\left(\mathbf{x}_{n}^{\star}, \mathbf{f}_{n}^{\star}\right)\right\}$, and a solution $\left(\mathbf{x}^{\star}, \mathbf{f}^{\star}\right)$ of $(98)$, such that

$$
\mathbf{f}_{n}^{\star} \rightarrow \mathbf{f}^{\star} \in \mathcal{Y}, \mathbf{x}_{n}^{\star} \rightarrow \mathbf{x}^{\star} \in \mathrm{V} \text { as } n \longrightarrow \infty .
$$

Proof. First, we denote by $\gamma: \mathrm{V} \longrightarrow \mathcal{Y}$ the operator $\mathbf{y} \longrightarrow\left\langle\iota \mathbf{y}, \zeta_{2} \mathbf{y}\right\rangle$, where $\iota: \mathrm{V} \longrightarrow L^{2}(\Omega)^{d}$ is the canonic embedding and $\rho_{2}: \mathrm{V} \longrightarrow L^{2}\left(T_{2}\right)^{d}$ is the restriction to the trace map 
to $\urcorner_{2}$. Next, we take the operators $\mathscr{N}: \mathrm{V} \times \mathrm{V} \longrightarrow \mathrm{V}, \mathscr{G}: \mathrm{V} \times \mathrm{V} \longrightarrow \mathrm{V}$, the function $\jmath: \mathrm{V} \times \mathrm{V} \longrightarrow \mathbb{R}$ and the element $\mathbf{f} \in \mathcal{Y}$ defined as follows:

$$
\begin{gathered}
\langle\mathscr{N}(\mathbf{x}, \mathbf{x}), \mathbf{y}\rangle_{\mathrm{V}}=\int_{\Omega} \mathscr{F}(\varepsilon(\mathbf{x}), \varepsilon(\mathbf{x})) \cdot \varepsilon(\mathbf{y}) d x+\int_{\urcorner_{3}} p\left(x_{v}\right) y_{v} d a, \\
\langle\mathscr{G}(\mathbf{x}, \mathbf{x}), \mathbf{y}\rangle_{\mathrm{V}}=\int_{\urcorner_{3}} q\left(x_{v}-\kappa\right) y_{v} d a, \\
\jmath: \mathcal{V} \times \mathcal{V} \longrightarrow \mathbb{R}, \quad \jmath(\mathbf{x}, \mathbf{y})=\mu \int_{\urcorner_{3}} p\left(x_{v}\right)\left\|\mathbf{y}_{\tau}\right\| d a, \\
\mathbf{f}=\left(\mathbf{f}_{0}, \mathbf{f}_{2}\right), \quad \forall \mathbf{x}, \mathbf{y} \in \mathrm{V} .
\end{gathered}
$$

Then it is clear that

$\mathbf{x} \in \mathscr{K}$ is a solution of (94) if and only if

$$
\langle\mathscr{N}(\mathbf{x}, \mathbf{x}), \mathbf{y}-\mathbf{x}\rangle_{\mathrm{V}}+\jmath(\mathbf{x}, \mathbf{y})-\jmath(\mathbf{x}, \mathbf{x}) \geq\langle\mathbf{f}, \gamma \mathbf{y}-\gamma \mathbf{x}\rangle_{\mathcal{Y}}, \forall y \in \mathscr{K} .
$$

For each $n \in \mathbb{N}, \mathbf{x}_{n} \in \hat{\mathscr{K}}$ is a solution of (107) if and only if

$$
\left\langle\mathscr{N}\left(\mathbf{x}_{n}, \mathbf{x}_{n}\right), \mathbf{y}-\mathbf{x}_{n}\right\rangle_{\mathrm{V}}+\frac{1}{\zeta_{n}}\left\langle\mathscr{G}\left(\mathbf{x}_{n}, \mathbf{x}_{n}\right), \mathbf{y}-\mathbf{x}_{n}\right\rangle_{\mathrm{V}}+\jmath\left(\mathbf{x}_{n}, \mathbf{y}\right)-\jmath\left(\mathbf{x}_{n}, \mathbf{x}_{n}\right) \geq\left\langle\mathbf{f}_{n}, \mathbf{y}-\mathbf{x}_{n}\right\rangle_{\mathcal{Y}}, \forall \mathbf{y} \in \hat{\mathscr{K}} .
$$

We can now continue with the proof of the two parts of the theorem.

(a) From the results of Sections 2 and 3. Assume that $\left.\mathcal{X}=\mathrm{V}, \mathcal{Y}=L^{2}(\Omega)^{d} \times L^{2}(\urcorner_{2}\right)^{d}, \mathscr{K}$ and $\hat{\mathscr{K}}$ defined by (93) and (104), respectively. $\mathscr{N}$ defined by (114), $\mathscr{G}$ defined by (115), $\jmath$ defined by (116) and $\mathbf{f}$ given by (117) and (7)-(15), (29)-(37) are hold.

Then from (82)-(84) we have

$$
\begin{aligned}
\langle\mathscr{N}(\mathbf{x}, \mathbf{x})-\mathscr{N}(\mathbf{y}, \mathbf{y}), \mathbf{x}-\mathbf{y}\rangle_{\mathrm{V}} & \geq-\alpha_{\mathscr{F}}\|\mathbf{x}-\mathbf{y}\|_{\mathrm{V}}^{2} \\
\langle\mathscr{N}(\mathbf{x}, \mathbf{x})-\mathscr{N}(\mathbf{y}, \mathbf{y}), \mathbf{x}-\mathbf{y}\rangle_{\mathrm{V}} & \leq-\beta_{\mathscr{F}}\|\mathbf{x}-\mathbf{y}\|_{\mathrm{V}^{\prime}}^{2} \\
\|\mathscr{N}(\mathbf{x}, \mathbf{x})-\mathscr{N}(\mathbf{y}, \mathbf{y})\| & \leq\left(\xi_{\mathscr{F}}+\varsigma_{\mathscr{F}}+d_{0}^{2} \xi_{p}\right)\|\mathbf{x}-\mathbf{y}\|_{\mathrm{V}}, \forall \mathbf{x}, \mathbf{y} \in \mathrm{V} .
\end{aligned}
$$

Hence, from conditions (8)-(9) hold with

$$
\beta_{\mathscr{N}}-\alpha_{\mathscr{N}}=\beta_{\mathscr{F}}-\alpha_{\mathscr{F}} .
$$

Obviously condition (11) is satisfied. On the other side, an elementary calculation depend on the definition (116) and the trace inequality (81) shows that

$$
\jmath\left(\mathbf{x}_{1}, \mathbf{y}_{2}\right)-\jmath\left(\mathbf{x}_{1}, \mathbf{y}_{2}\right)+\jmath\left(\mathbf{x}_{2}, \mathbf{y}_{1}\right)-\jmath\left(\mathbf{x}_{2}, \mathbf{y}_{2}\right) \leq d_{0}^{2} \mu \xi_{p}\left\|\mathbf{x}_{1}-\mathbf{x}_{2}\right\|_{\mathrm{V}}\left\|\mathbf{y}_{1}-\mathbf{y}_{2}\right\|_{\mathrm{V}}, \forall \mathbf{x}_{1}, \mathbf{x}_{2}, \mathbf{y}_{1}, \mathbf{y}_{2} \in \mathrm{V}
$$

Therefore, condition (12) holds with $\alpha_{j}=d_{0}^{2} \mu \xi_{p}$ and also from $\lambda=0$ and $\rho=d_{0}^{2} \mu \xi_{p}$ the condition (22) holds. Using (90), it also satisfy the smallness conditions (13) and (23). The conditions (24)-(26) arise from standard compactness arguments and, finally, assumptions (36)-(37) are the direct consequence of the definitions (115), (104) and (93) along with the properties (99)-(103) of the function $q$.

Combining (118),(119) with (111)), we arrived the proof of the theorem.

(b) we utilize the results of Sections 2 and 4 in the functional framework already described above, with the functionals $\mathscr{L}$ and $\mathscr{L}_{n}$ given by (97) and (109), respectively. It is clear to see that in this circumstance the assumptions (7)-(13), (15)-(26), (29)-(32), $(17)_{n}-(19)_{n},(20)_{n}-(21)_{n}$ and (57)-(61) hold with an appropriate choice of the functions $\mathscr{B}, \mathscr{D}, \mathscr{B}_{n}$ and $\mathscr{D}_{n}$. Therefore, we are in a position to utilize Lemma 2 in order to determine the existence of a solution of the optimal control problems in (98) and (109). Applying the Theorem 4 to prove the convergence (113). 


\section{A Stationary Heat Transfer Problem with Unilateral Constraints}

Applying the abstract results of Sections 2 and 4, in this section we describes a heat transfer boundary value problem. The classical formulation of the following problem for finding a temperature field $x: \Omega \longrightarrow \mathbb{R}$ such that

$$
\begin{gathered}
x \geq 0, \Delta x+f \leq 0, \quad x(\Delta x+f)=0 \quad \text { a.e. in } \Omega, \\
x=0, \text { a.e. in }\urcorner_{1}, \\
x=b, \text { a.e. in }\rceil_{2}, \\
\left.-\frac{\partial x}{\partial v}=q, \text { a.e. in }\right\rceil_{3} .
\end{gathered}
$$

Here, $\Omega$ is a bounded domain in $\mathbb{R}^{d}(d=1,2,3)$ with smooth boundary $\partial \Omega=\rceil_{1} \cup T_{2} \cup T_{3}$ and outer normal unit $v$. Assume that $\rceil_{1}, 7_{2}, 7_{3}$ are disjoint measurable sets and, moreover, meas $\left(T_{1}\right)>0$. We do not mention the dependence of the different functions on the spatial variable $\mathbf{x} \in \Omega \cup \partial \Omega$. Let $f$ be a internal energy function, $b$ be the prescribed temperature field on $\tau_{2}$ and $q$ be the heat flux prescribed on $\rceil_{3}$. Furthermore, $\frac{\partial x}{\partial y}$ denotes the normal derivative of $x$ on $7_{3}$.

For the variational analysis of (122)-(125) we consider the space

$$
\left.\mathrm{V}=\left\{\mathbf{y} \in H^{1}(\Omega): \mathbf{y}=0 \text { on }\right\rceil_{1}\right\}
$$

Let $\left(\mathrm{V},\langle\cdot, \cdot\rangle_{\mathrm{V}}\right)$ be a real Hilbert space. Assume that

$$
f \in L^{2}(\Omega), \quad b \in L^{2}\left(T_{2}\right), q \in L^{2}\left(\top_{3}\right),
$$

there exists $y_{0} \in \mathrm{V}$ such that

$$
\left.0 \leq y_{0} \in \Omega \text { and } y_{0}=b \text { on }\right\rceil_{2}
$$

We introduce the set

$$
\mathscr{K}=\left\{y \in \mathrm{V}: y \geq 0 \text { on } \Omega, y=b \text { on } \mathrm{T}_{2}\right\} .
$$

Then, the variational formulation of Equations (122)-(125), to obtained through standard arguments for finding $x \in \mathscr{K}$ such that

$$
\int_{\Omega} \nabla x \cdot(\nabla y-\nabla x) d x+\int_{\urcorner_{3}} q(y-x) d a \geq \int_{\Omega} f(y-x) d x, \forall y \in \mathscr{K} .
$$

Now, we introduce the set of admissible pairs for inequality (129) defined by

$$
\mathcal{V}_{a d}^{t}=\left\{(x, f) \in \mathscr{K} \times L^{2}(\Omega) \text { such that (129) holds }\right\} .
$$

Moreover, we consider two constants $\omega, \omega$ and a function $\psi$ such that

$$
\omega>0, \omega>0, \psi \in L^{2}(\Omega) \text {. }
$$

Now we associate to (129) with above data, for finding optimal control problem $\left(x^{\star}, f^{\star}\right) \in \mathcal{V}_{a d}^{t}$ such that

$\omega \int_{\Omega}\left(x^{\star}-\psi\right)^{2} d x+\omega \int_{\Omega}\left(f^{\star}\right)^{2} d x=\min _{(x, f) \in \mathcal{V}_{a d}^{t}}\left\{\omega \int_{\Omega}(x-\psi)^{2} d x+\omega \int_{\Omega} f^{2} d x\right\}$.

Next, we introduce the set

$$
\hat{\mathscr{K}}=\{y \in \mathrm{V}: y \geq 0 \text { in } \Omega\} .
$$


For each $n \in \mathbb{N}$, we assume that the functions $f_{n}, \psi_{n}$ and the constants $\zeta_{n}, \omega_{n}, \omega_{n}$, are given and satisfy the following conditions:

$$
\begin{gathered}
f_{n} \in L^{2}(\Omega), \\
\zeta_{n}>0, \omega_{n}>0, \omega_{n}>0, \psi_{n} \in L^{2}(\Omega) .
\end{gathered}
$$

Then, for each $n \in \mathbb{N}$, we consider the following perturbation of (129) for finding $x_{n} \in \hat{\mathscr{K}}$ such that

$$
\int_{\Omega} \nabla x_{n} \cdot\left(\nabla y-\nabla x_{n}\right) d x+\int_{\urcorner_{3}} q\left(y-x_{n}\right) d a+\frac{1}{\zeta_{n}} \int_{\urcorner_{2}}\left(x_{n}-b\right)\left(y-x_{n}\right) d a \geq \int_{\Omega} f_{n}\left(y-x_{n}\right) d x, \forall y \in \hat{\mathscr{K}} .
$$

It is easy to see that (136) represents the variational formulation of the following boundary value problem for finding a temperature field $x_{n}: \Omega \longrightarrow \mathbb{R}$ such that

$$
\begin{gathered}
x_{n} \geq 0, \Delta x_{n}+f_{n} \leq 0, \quad x_{n}\left(\Delta x_{n}+f_{n}\right)=0 \text { a.e. in } \Omega, \\
\left.x_{n}=0 \text { a.e. on }\right\urcorner_{1}, \\
\left.-\frac{\partial x_{n}}{\partial v}=\frac{1}{\zeta_{n}}\left(x_{n}-b\right) \text { a.e. on }\right\urcorner_{2}, \\
\left.-\frac{\partial x_{n}}{\partial v}=q \text { a.e. on }\right\urcorner_{3},
\end{gathered}
$$

The set of admissible pairs for inequality (136) is defined by

$$
\mathcal{V}_{a d}^{t n}=\left\{\left(x_{n}, f_{n}\right) \in \hat{\mathscr{K}} \times L^{2}(\Omega) \text { such that (136) holds }\right\} .
$$

Furthermore, the associated optimal control problem for finding $\left(x_{n}^{\star}, f_{n}^{\star}\right) \in \mathcal{V}_{a d}^{t n}$ such that

$$
\omega_{n} \int_{\Omega}\left(x_{n}^{\star}-\psi_{n}\right)^{2} d x+\omega_{n} \int_{\Omega}\left(f_{n}^{\star}\right)^{2} d x=\min _{(x, f) \in \mathcal{V}_{a d}^{t n}}\left\{\omega_{n} \int_{\Omega}\left(x-\psi_{n}\right)^{2} d x+\omega_{n} \int_{\Omega} f^{2} d x\right\} .
$$

Theorem 6. Assume that (126)-(127), (131), (134) and (135) hold. Then

(a) problem (129) has a unique solution and, for each $n \in \mathbb{N}$ the problem (136) has a unique solution also. Furthermore, if

$$
\zeta_{n} \longrightarrow 0 \text { and } f_{n} \rightarrow f \in L^{2}(\Omega) \text { as } n \longrightarrow \infty,
$$

the solution of problem (136) converges to the solution of problem (129), i.e.,

$$
x_{n} \longrightarrow x \in \mathrm{V} \text { as } n \longrightarrow \infty .
$$

(b) problem (132) has at least one solution and, for each $n \in \mathbb{N}$, problem (142) has at least one solution. Moreover, the solution of problem (132) is unique if

$$
\psi=0_{L^{2}(\Omega)}
$$

and, for each $n \in \mathbb{N}$, the solution of (142) is unique, if

$$
\psi_{n}=0_{L^{2}(\Omega)}
$$

(c) Assume that

$$
\zeta_{n} \rightarrow 0, \omega_{n} \rightarrow \omega, \omega_{n} \rightarrow \infty, \psi_{n} \rightarrow \psi \in L^{2}(\Omega) \text { as } n \longrightarrow \infty,
$$


and let $\left\{\left(x_{n}^{\star}, f_{n}^{\star}\right)\right\}$ be a sequence of solutions of (142). Then, there exists a subsequence of the sequence $\left\{\left(x_{n}^{\star}, f_{n}^{\star}\right)\right\}$, again denoted by $\left\{\left(x_{n}^{\star}, f_{n}^{\star}\right)\right\}$, and a solution $\left(x^{\star}, f^{\star}\right)$ of $(132)$, such that

$$
f_{n}^{\star} \rightarrow f^{\star} \in L^{2}(\Omega), x_{n}^{\star} \rightarrow x^{\star} \in V \text { as } n \longrightarrow \infty .
$$

Moreover, if $\psi=0_{L^{2}(\Omega)}$, then the whole sequence $\left\{\left(x_{n}^{\star}, f_{n}^{\star}\right)\right\}$ satisfies (146) where $\left(x^{\star}, f^{\star}\right)$ represents the unique solution of (132).

Proof. To begin, we will introduce some notation that will allow us to write the problem in an equivalent way. To this end, Let $\gamma: \mathrm{V} \longrightarrow L^{2}(\Omega)$ be the canonical inclusion of $\mathrm{V}$ in $L^{2}(\Omega)$. Moreover, we consider the operators $\mathscr{N}: \mathrm{V} \times \mathrm{V} \longrightarrow \mathrm{V}, \mathscr{G}: \mathrm{V} \times \mathrm{V} \longrightarrow \mathrm{V}$ defined by

$$
\begin{gathered}
\langle\mathscr{N}(x, x), y\rangle_{\mathrm{V}}=\int_{\Omega} \nabla x \cdot \nabla y d x+\int_{\urcorner_{3}} q y d a, \forall x, y \in \mathrm{V}, \\
\langle\mathscr{G}(x, x), y\rangle_{\mathrm{V}}=\int_{\urcorner_{2}}(x-b) y d a, \forall x, y \in \mathrm{V} .
\end{gathered}
$$

Then, $x$ is a solution of (129) if and only if

$$
x \in \mathscr{K},\langle\mathscr{N}(x, x), y-x\rangle_{\mathrm{V}} \geq\langle f, y-x\rangle_{L^{2}(\Omega)}, \forall y \in \mathscr{K} .
$$

Moreover, for each $n \in \mathbb{N}, x_{n} \in \hat{\mathscr{K}}$ is a solution of (136) if and only if

$$
\langle\mathscr{N}(x, x), y-x\rangle_{\mathrm{V}}+\frac{1}{\zeta_{n}}\left\langle\mathscr{G}\left(x_{n}, x_{n}\right), y-x_{n}\right\rangle_{\mathrm{V}} \geq\left\langle f_{n}, y-x_{n}\right\rangle_{L^{2}(\Omega)}, \forall y \in \hat{\mathscr{K}}
$$

Next, denote by $\mathscr{L}: \mathrm{V} \times L^{2}(\Omega) \longrightarrow \mathbb{R}$ and $\mathscr{L}_{n}: \mathrm{V} \times L^{2}(\Omega) \longrightarrow \mathbb{R}$ the cost functionals given by

$$
\begin{gathered}
\mathscr{L}(x, f)=\omega\|x-\psi\|_{L^{2}(\Omega)}^{2}+\omega\|f\|_{L^{2}(\Omega)^{\prime}}^{2} \\
\mathscr{L}_{n}(x, f)=\omega_{n}\left\|x-\psi_{n}\right\|_{L^{2}(\Omega)}^{2}+\omega_{n}\|f\|_{L^{2}(\Omega)^{\prime}}^{2} \quad \forall(x, f) \in \mathrm{V} \times L^{2}(\Omega) .
\end{gathered}
$$

Then, it is clear to see that $\left(x^{\star}, f^{\star}\right)$ is a solution of (132) if and only if

$$
\left(x^{\star}, f^{\star}\right) \in \mathcal{V}_{a d}^{t} \quad \text { and } \mathscr{L}\left(x^{\star}, f^{\star}\right)=\min _{\left(x^{\star}, f^{\star}\right) \in \mathcal{V}_{a d}^{t}} \mathscr{L}(x, f) .
$$

Moreover, for each $n \in \mathbb{N},\left(x_{n}^{\star}, f_{n}^{\star}\right)$ is a solution of (132) if and only if

$$
\left(x_{n}^{\star}, f_{n}^{\star}\right) \in \mathcal{V}_{a d}^{t n} \quad \text { and } \mathscr{L}_{n}\left(x_{n}^{\star}, f_{n}^{\star}\right)=\min _{\left(x^{\star}, f^{\star}\right) \in \mathcal{V}_{a d}^{t n}} \mathscr{L}_{n}(x, f) .
$$

We now proceed with the proof of the two parts of the theorem.

(a) Using the abstract results of Sections 2 and 3 with $\mathcal{X}=\mathrm{V}, \mathcal{Y}=L^{2}(\Omega)$, $\mathscr{K}$ and $\hat{\mathscr{K}}$ defined by (128) and (133), respectively, and $\mathscr{N}$ defined by (147), $\mathscr{G}$ defined by (148), and $\jmath \equiv 0$, and conditions (7)-(15), (29)-(37) are satisfied. Therefore, we are in a position to apply Theorem 1 and Lemma 1 in order to deduce the existence of a unique solution of the variational inequalities in (149) and (150), respectively and also by Theorem 3 to obtain the convergence (144). Combining (149) and (150), we arrived the conclusion to the proof of the statement (a) in Theorem 6.

(b) We employ the Theorem 2 and Theorem 4 with the functionals $\mathscr{L}$ and $\mathscr{L}_{n}$ given by (151) and (152), respectively, and conditions (7)-(13), (15)-(26), (29)-(32), (17) $n-(19)_{n}$, $(20)_{n}-(21)_{n},(57)$ and (58)-(59) are valid. As a result, we can clearly see that using Theorem 2 and Lemma 2, we can conclude the existence of a solution to the optimal control problems in (153) and (154), respectively. 
The uniqueness of the solution to the problem (132) when

$$
\psi=0_{L^{2}(\Omega)}
$$

follows from a strict convexity argument.

For any $f \in L^{2}(\Omega)$, let $x(f)$ denote the solution of the variational inequality in (149). Then, in [32], it was demonstrated that the functional

$$
f \longmapsto \mathscr{L}(x(f), f)=\omega\|x(f)\|_{L^{2}(\Omega)}^{2}+\omega\|f\|_{L^{2}(\Omega)}^{2}
$$

is strictly convex. Hence, the optimal control problem in (153) has a unique solution and the uniqueness of the solution of (142) in the case $\psi_{n}=0_{L^{2}(\Omega)}$ follows from the same argument. Hence, combined with the equivalence results (153) and (154) allows us to conclude the proof of the (b) in Theorem 6.

(c) The convergence (146) is a direct consequence of Theorem 4 . The convergence (146) of the whole sequence $\left\{\left(x_{n}^{\star}, f_{n}^{\star}\right)\right\}$ in the case $\psi=0_{L^{2}(\Omega)}$ follows from a standard argument, since in this case (132) has a unique solution.

Author Contributions: Conceptualization, S.-S.C., A.A.H.A., S., M.L. and J.T.; methodology, S.-S.C., A.A.H.A., S., M.L. and J.T.; software, S.-S.C., A.A.H.A., S., M.L. and J.T.; validation, S.-S.C., A.A.H.A., S., M.L. and J.T.; writing-original draft preparation, S.-S.C., A.A.H.A., S., M.L. and J.T.; writing-review and editing, S.-S.C., A.A.H.A., S., M.L. and J.T.; visualization, S.-S.C., A.A.H.A., S., M.L. and J.T.; supervision, S.-S.C., A.A.H.A., S., M.L. and J.T.; project administration, S.-S.C., A.A.H.A., S., M.L. and J.T.; funding acquisition, S.-S.C., A.A.H.A., S., M.L. and J.T. All authors have read and agreed to the published version of the manuscript.

Funding: This work was supported by the Scientific Research Fund of Science and Technology Department of Sichuan Provincial(2018JY0340,2018JY0334) and supported by the Natural Scientific Fund of Center for General Education, China Medical University, Taichung, Taiwan.

Institutional Review Board Statement: Not applicable.

Informed Consent Statement: Not applicable.

Data Availability Statement: The data sets used and/or analysed during the current study are available from the corresponding author on reasonable request.

Acknowledgments: The authors wish to express their gratitude supported by the Center for General Education, China Medical University, Taichung, Taiwan to the editor and referees for careful reading of the manuscript, and their valuable comments and suggestions.

Conflicts of Interest: The authors declare that they have no conflict of interest to this work.

\section{References}

1. Mei, D.; Sun, Y.; Zhao, H.; He, X.T. A closed-form solution for the boundary value problem of gas pressurized circular membranes in contact with frictionless rigied plates. Mathematics 2020, 8, 1017. [CrossRef]

2. Zvyagin, V.; Zvyagin, A.; Ustiuzhaninova, A. Optimal feedback control problem for the fractional Voigt- $\alpha$ model. Mathematics 2020, 8, 1197. [CrossRef]

3. Duvaut, G.; Lions, J.-L. Inequalities in Mechanics and Physics; Springer: Berlin/Heidelberg, Germany, 1976.

4. Barbu, V.; Korman, P. Analysis and Control of Nonlinear Infinite Dimensional Systems; Academic Press: Boston, MA, USA, 1993.

5. Matei, A.; Micu, S.; Nita, C. Optimal control for antiplane frictional contact problems involving nonlinearly elastic materials of Hencky type. Math. Mech. Solids 2018, 23, 308-328. [CrossRef]

6. Baiocchi, C.; Capelo, A. Variational and Quasivariational Inequalities: Applications to Free-Boundary Problems; John Wiley: Chichester, UK, 1984.

7. Clarke, F.H. Optimization and Nonsmooth Analysis; Wiley: New York, NY, USA, 1983.

8. Brezis, H. Functional Analysis, Sobolev Spaces and Partial Differential Equations; Springer: New York, NY, USA, 2011.

9. Naniewicz, Z.; Panagiotopoulos, P.D. Mathematical Theory of Hemivariational Inequalities and Applications; Marcel Dekker, Inc.: New York, NY, USA, 1995.

10. Kim, J.K. Salahuddin: Local sharp vector variational type inequality and optimization problems. Mathematics 2020, 8, 1844. [CrossRef] 
11. Migórski, S.; Ochal, A.; Sofonea, M. Nonlinear Inclusions and Hemivariational Inequalities. Models and Analysis of Contact Problems. In Advances in Mechanics and Mathematics; Springer: New York, NY, USA, 2013.

12. Motreanu, D.; Sofonea, M. Quasi variational inequalities and applications in frictional contact problems with normal compliance. Adv. Math. Sci. Appl. 2000, 10, 103-118.

13. Salahuddin. The existence of solution for equilibrium problems in Hadamard manifolds. Trans. Razmadze Math. Inst. 2017, 171, 381-388. [CrossRef]

14. Barbu, V. Optimal Control of Variational Inequalities; Research Notes in Mathematics; 100 Pitman: Boston, MA, USA, 1984. [CrossRef]

15. Bonnans, J.F.; Tiba, D. Pontryagin's principle in the control of semilinear elliptic variational inequalities. Appl. Math. Optim. 1991, 23, 299-312. [CrossRef]

16. Mignot, F.; Puel, J.-P. Optimal control in some variational inequalities. SIAM J. Control Optim. 1984, 22, 466-476. [CrossRef]

17. Peng, Z.; Kunisch, K. Optimal control of elliptic variational-hemivriational inequalities. J. Optim. Theory Appl. 2018, 178, 1-25. [CrossRef]

18. Capatina, A. Variational Inequalities Frictional Contact Problems. In Advances in Mechanics and Mathematics; Springer: New York, NY, USA, 2014; Volume 31.

19. Eck, C.; Jarusek, J.; Krbec, M. Unilateral Contact Problems: Variational Methods and Existence Theorems. In Pure and Applied Mathematics; Chapman/CRC Press: New York, NY, USA, 2005; Volume 270.

20. Han, W.; Sofonea, M. Quasistatic Contact Problems in Viscoelasticity and Viscoplasticity. In Studies in Advanced Mathematics; American Mathematical Society, Providence, RI-International Press: Somerville, MA, USA, 2002; Volume 30.

21. Boukrouche, M.; Tarzia, D.A. Convergence of distributed optimal control problems governed by elliptic variational inequalities. Comput. Optim. Appl. 2012, 53, 375-393. [CrossRef]

22. Capatina, A. Optimal control of Signorini problem. Numer. Funct. Anal. Optim. 2000, 21, 817-828. [CrossRef]

23. Matei, A.; Micu, S. Boundary optimal control for nonlinear antiplane problems. Nonlinear Anal. Theory Methods Appl. 2011, 74, 1641-1652. [CrossRef]

24. Liu, Z.H.; Migorski, S.; Zeng, S.D. Partial differential variational inequalities involving nonlocal boundary conditionsin Banach spaces. J. Differ. Equ. 2017, 263, 3989-4006. [CrossRef]

25. Matei, A.; Micu, S. Boundary optimal control for a frictional contact problem with normal compliance. Appl. Math. Optim. 2018, 78, 379-401. [CrossRef]

26. Sofonea, M.; Xiao, Y.B. Boundary optimal control of a nonsmooth frictionless contact problem. Comput. Math. Appl. 2019, 78, 152-165. [CrossRef]

27. Kim, J.K.; Salahuddin; Lim, W.H. An iterative algorithm for generalized mixed equilibrium problems and fixed points of nonexpansive semigroups. J. Appl. Math. Phys. 2017, 5, 276-293. [CrossRef]

28. Mewomo, O.T.; Oyewole, O.K. An iterative approximation of common solutions of split generalized vector mixed equilibrium problem and some certain optimization problems. Demonstr. Math. 2021, 54, 335-358. [CrossRef]

29. Tuyen, T.M.; Hammad, H.A. Effect of shrinking projection and CQ-methods on two inertial forward-backward algorithms for solving variational problems. Rendiconti del Circolo Matematico di Palermo Series 2 2021, 70, 1669-1683. [CrossRef]

30. Salahuddin. Perturbation strategy for splitting operator method to solve the set-valued variational inequalities. J. Math. Inequal 2021, 15, 117-130. [CrossRef]

31. Salahuddin. The extragradient method for quasi monotone variational inequalities. Optimization 2021, 70, 127-136. [CrossRef]

32. Boukrouche, M.; Tarzia, D.A. Existence, uniqueness and convergence of optimal control problems associated with parabolic variational inequalities of the second kind. Nonlinear Anal. Real World Appl. 2011, 12, 2211-2224. [CrossRef]

33. Hlavacek, I.; Haslinger, J.; Necas, J.; Lovisek, J. Solution of Variational Inequalities in Mechanics; Springer: New York, NY, USA, 1988.

34. Panagiotopoulos, P.D. Inequality Problems in Mechanics and Applications; Birkhauser: Boston, MA, USA, 1985.

35. Ahmad, R.; Kazmi, K.R.; Salahuddin. Completely generalized nonlinear variational inclusion involving relaxed Lipschitz and relaxed monotone mappings. Nonlinear Anal. Forum. 2000, 5, 61-69.

36. Sofonea, M. Optimal Control of Variational Inequalities with Applications to Contact Mechanics. In Current Trends in Mathematical Analysis and Its Interdisciplinary Applications; Dutta, H., Ed.; Springer Nature: Basel, Switzerland, 2019; Chapter 13, pp. $443-487$.

37. Kikuchi, N.; Oden, J.T. Contact Problems in Elasticity: A Study of Variational Inequalities and Finite Element Methods; SIAM: Philadelphia, PA, USA, 1988.

38. Sofonea, M.; Matei, A. Mathematical Models in Contact Mechanics; London Mathematical Society Lecture Note Series; Cambridge University Press: Cambridge, UK, 2012; Volume 398

39. Sofonea, M.; Tarzia, D.A. Convergence results for optimal control problems governed by elliptic quasivariational inequalities. Num. Funct. Anal. Optim. 2020, 41, 1326-1351. [CrossRef]

40. Sofonea, M.; Migorski, S. Variational-hemivariational Inequalities with Applications. In Pure and Applied Mathematics; Chapman and Hall/CRC Press: Boca Raton, MA, USA, 2018.

41. Lee, B.S.; Salahuddin. Solutions for general class of hemivariational like inequality systems. J. Nonlinear Convex Anal. 2015, $16,141-150$. 